\title{
A COMPONENTE MENTAL: UM ASPETO POSITIVO DA QUALIDADE DE VIDA DE UMA POPULAÇÃO \\ | Ana Guerra ${ }^{1}$; Ana Silva ${ }^{2}$; Clementina Sousa ${ }^{3}$; Luísa Ferreira ${ }^{4}$; Helena Carvalho ${ }^{5}$; Sandra Silva ${ }^{6}$ |
}

\section{RESUMO}

Saúde é uma dimensão importante da Qualidade de Vida (QV), que integra outros domínios para além da Saúde. Perante esta perspetiva, os profissionais de saúde orientam a sua prática com vista à prestação de cuidados que visam proporcionar às pessoas o máximo bem-estar e QV ao longo de todo o ciclo vital.

Uma melhor QV constitui um dos objetivos mais ambicionados pela generalidade dos seres humanos representando, deste modo, uma crescente relevância na prática clínica de Enfermagem.

Neste sentido constitui-se objetivo do estudo avaliar a perceção da QV da população de Paredes de Coura, no ano 2015.

Para tal desenhou-se um estudo quantitativo, de natureza descritiva, com uma amostra de 447 indivíduos selecionada pelo método de amostragem probabilística e aleatória estratificada. Como instrumento de recolha de dados utilizou-se um questionário de caracterização sociodemográfica e o questionário do Estado de Saúde (MOS SF-36v2).

Os resultados evidenciam que a população de Paredes de Coura apresenta uma melhor perceção de bem-estar psicológico e social; as piores perceções dizem respeito à Vitalidade e Saúde Geral. A maioria da população refere ausência de dor e de limitações associadas à mesma, sendo a perceção global de QV tendencialmente positiva.

Em conclusão, a população de Paredes de Coura apresenta uma perceção de QV positiva, destacando-se a Componente Mental.

\section{PALAVRAS-CHAVE: Saúde; Promoção de Saúde; Qualidade de Vida; Enfermagem em Saúde Comunitária}

\section{RESUMEN}

“Componente Mental: un aspecto positivo en la calidad de vida de una población"

La Salud es una dimensión importante de Calidad de Vida (CV), que integra otras áreas distintas de la Salud. Ante esta perspectiva, profesionales de la salud guían su práctica con el fin de proporcionar el cuidado que tienen como objetivo dar a las personas el máximo de bienestar y calidad de vida a lo largo del ciclo de vida.

Una mejor calidad de vida es uno de los objetivos más ambiciosos de la mayoría de los seres humanos, lo que constituye un aumento de relevancia en la práctica clínica de la Enfermería.

En este sentido, constituye objetivo del estudio para evaluar la percepción de la calidad de vida de la población de Paredes de Coura en 2015.

Para ello se diseñó un estudio cuantitativo, descriptivo, con una muestra de 447 individuos seleccionados por el método de muestreo aleatorio probabilístico y estratificado. Como instrumento de recolección de datos se utilizó el cuestionario socio demográfico y el cuestionario del Estado de Salud (MOS SF-36v2).

Los resultados muestran que la población de Paredes de Coura tiene una mejor percepción de bienestar psicológico y social; las peores percepciones se relacionan con la Vitalidad y la Salud Mental General. La mayor parte de la población informó de ausencia de dolor y limitaciones asociadas con el mismo, siendo atendida la percepción positiva en general la calidad de vida.

En conclusión, la población de Paredes de Coura tiene una percepción de la CV positiva, destacando el componente mental.

\section{DESCRIPTORES: Salud; Promoción de la Salud; Calidad de Vida; Enfermería en Salud Comunitaria}

\section{ABSTRACT \\ "The Mental component: a positive aspect of the Quality of Life of a population"}

Health is an important dimension of Quality of Life (QoL), that also integrates other aspects besides health. In this line of perspective, health professionals conduct their practice with the aim of providing their patients with the care that assures the maximum well-being and life's quality during all their life.

A better QoL is one of the most ambitioned objectives by the generality of human beings, having, this way, a growing relevance in Nursing clinical practice.

Therefore, the objective of the study is to understand the perception of QoL of the individuals of Paredes de Coura, in the year of 2015.

A quantitative study was designed, of descriptive nature, with a sample of 447 individuals, selected by the method of probabilistic and stratified random sampling. As data collection instrument it was used a questionnaire of sociodemographic characterization and the questionnaire of State of Health (MOS SF-36v2).

The results evidence that the population of Paredes de Coura presents the better perception in relation to psychological and social well-being parameters; the worst perceptions are related to Vitality and General Health. Most of the population refers absence of pain and associated limitations, being the global perception of QoL tendentiously positive.

Concluding, the population of Paredes de Coura shows a positive QoL perception, with a highlight in the Mental Component.

\section{KEYWORDS: Health; Health Promotion; Quality of Life; Nurs- ing in Community Health}

Submetido em 31-01-2017

Aceite em 30-06-2017

1 Enfermeira; Licenciatura em Enfermagem, Escola Superior de Saúde - Instituto Politécnico de Viana do Castelo

2 Enfermeira; Licenciatura em Enfermagem, Escola Superior de Saúde - Instituto Politécnico de Viana do Castelo.

3 Professor-Adjunto, Escola Superior de Saúde - Instituto Politécnico de Viana do Castelo. Mestrado em Enfermagem, clementinasousa@ess.ipvc.pt

4 Enfermeira; Licenciatura em Enfermagem, Escola Superior de Saúde - Instituto Politécnico de Viana do Castelo

5 Enfermeira; Licenciatura em Enfermagem, Escola Superior de Saúde - Instituto Politécnico de Viana do Castelo

6 Enfermeira; Licenciatura em Enfermagem, Escola Superior de Saúde - Instituto Politécnico de Viana do Castelo

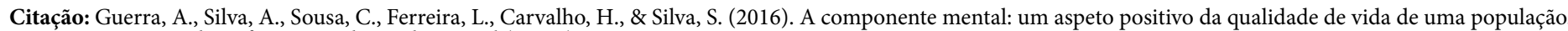
Revista Portuguesa de Enfermagem de Saúde Mental (Spe. 5), 75-80. 


\section{INTRODUÇÃO}

A saúde revela-se um conceito amplo e holístico, na medida em que engloba a pessoa nas suas múltiplas dimensões, estando profundamente ligada com o modo de viver da população, sendo influenciada pelo conhecimento, ambiente físico, social, económico e cultural em que os indivíduos se inserem (Lourenço, 2013). Além disso, implica bem-estar, isto é, as pessoas referem ter saúde quando se sentem bem e capazes de realizar as atividades de vida diária. $\mathrm{O}$ bem-estar abrange também a existência de trabalho, habitação e, igualmente, a satisfação das necessidades básicas, de alimentação, de educação, da afetividade e perspetivas positivas para o futuro. Segundo Praça (2012 citando Albuquerque e Matos, 2006) a saúde é um desafio, um conceito dinâmico difícil de definir e medir, podendo ser entendida como uma dimensão da QV. Esta é promovida por acontecimentos positivos que contribuem para a QV, partindo dos contextos de proximidade e das relações imediatas do indivíduo com o meio envolvente.

A forma como o indivíduo se posiciona nestes contextos e nas interações consigo próprio e com o meio envolvente depende das suas características pessoais, cognitivas e comportamentais e emocionais e físicas (Gonçalves, 2008 citando Parreira, 2006). Assim, a QV representa uma perceção individual que é influenciada, de forma significativa por estes determinantes, daí que seja considerada um constructo eminentemente humano.

Assim como a saúde, a QV é também um conceito difícil de definir considerando o seu caráter subjetivo, inter-relacional e multidimensional (Zamberlan, Calvetti, Deisvaldi e De Siqueira, 2010). Frequentemente são utilizados como sinónimos apesar de possuírem diferentes conceções, contudo reconhece-se a relação existente entre si. A saúde é portanto encarada como um dos domínios mais importantes da QV, sendo que uma mudança no estado de saúde de um indivíduo pode-se constituir significativa para a perceção de QV do mesmo (André, 2005; Martins, 2006), ou seja o estado de saúde pode influenciar a perceção de QV de um indivíduo.

Neste sentido surgiu a importância de se avaliar a perceção de QV de várias populações, onde vários autores tiraram as suas inferências, nomeadamente Coelho e Pedroso (2012) que concluíram que a dimensão Função Social (FS) e Desempenho Emocional (DE) obtiveram melhores resultados.
Já o pior resultado diz respeito à Dor Corporal (DC) Estes autores, a par de Almeida (2013), referem que, nos indivíduos acima dos 65 anos, os valores tendem a ser mais positivos na Componente Mental do que na Física. Um estudo de Fernandes (2014), no que diz respeito ao concelho de Paredes de Coura, os domínios SG e VT são os que apresentam os valores mais baixos, ao contrário da FS e DE que apresentam os valores mais elevados. A SM apresenta valores superiores a $70 \%$. No estudo de Correia (2012), com uma amostra constituída apenas por idosos, constatou-se que as dimensões com valores mais baixos foram a Saúde Geral (SG) e a Vitalidade (VT), a par das dimensões FS e DE que são de avaliação muito positiva. Num estudo de Ayama, Rodrigues, Silva e Feriancic (2009), com uma amostra exclusivamente constituída por octogenários os resultados mais elevados são na dimensão FS e os valores mais baixos pertencem à Componente Física. Braga et al. (2011) e Praça (2012) acrescentam-nos que a perceção de QV diminui com a idade, o que poderá justificar-se com as afirmações de Fechine \& Trompieri, em 2012 (Sousa, 2013) que nos dizem que inerente ao processo de envelhecimento natural, ocorre um conjunto de alterações que levam a um declínio funcional que afeta vários órgãos e sistemas, debilitando o organismo, nomeadamente o sistema musculosquelético.

No panorama nacional, o Inquérito Nacional de Saúde de 2005 e 2006, revela que $48,6 \%$ da população residente avalia a sua QV como boa ou muito boa e apenas $44,5 \%$ como nem má, nem boa (Instituto Nacional de Estatística [INE], 2009). De referir ainda que segundo os Censos 2011, pouco mais de metade $(51,23 \%)$ da população de Paredes de Coura apresentava idades compreendidas entre os 25 e os 64 anos, sendo esta a faixa etária mais representativa, seguida da faixa etária dos 65 ou mais anos com 26,88\% (INE, 2012).

A nível municipal, o $2^{\circ}$ e $3^{\circ}$ Inquérito Municipal de Saúde (IMS), realizados em freguesias do Município de Viana do Castelo, a maioria da população classifica a sua QV como boa ou muito boa (Inquérito Municipal de Saúde [IMS], 2011; IMS, 2012a).

No sentido destes vários estudos, pretendeu-se com este responder à seguinte questão de investigação "Qual a perceção de Qualidade de Vida da População de Paredes de Coura", com o intuito de contribuir para a definição de estratégias de intervenção em saúde e, designadamente, em Enfermagem mais adequadas às reais necessidades da população, promovendo, assim, a melhoria de cuidados e contribuindo para a criação do Perfil de Saúde do Município de Paredes de Coura. 
Partindo destes pressupostos, traçou-se como objetivo geral avaliar a perceção da QV da população de Paredes de Coura no ano de 2015.

\section{METODOLOGIA}

Com o intuito de dar resposta ao objetivo, optou-se por um estudo do tipo descritivo, inscrito no paradigma quantitativo. Constituiu a população alvo do estudo, a população de 8 freguesias representativas das 21 que integram o concelho de Paredes de Coura, onde residem cerca de 2272 indivíduos, dos quais foi extraída uma amostra pelo método de amostragem probabilístico e aleatório estratificado.

Definiu-se uma amostra de 603 indivíduos, considerando como critérios de inclusão: ser residente em Paredes de Coura e responder voluntariamente ao questionário. Para a avaliação da QV foi considerada uma amostra de 447 indivíduos, por se considerar também como critérios de inclusão: ser o próprio a responder e ter idade igual ou superior a 15 anos.

Os instrumentos de colheita de dados foram: um questionário de caracterização sociodemográfica e o questionário do Estado de Saúde (MOS SF-36v2), desenvolvido por John Ware e traduzido e validado para a população portuguesa por Ferreira (1998). O questionário é constituído por 36 itens distribuídos por oito dimensões. A Função Física (FF), o Desempenho Físico (DF), a DC, e a SG constituem a Componente Física. Por sua vez, a VT, a FS, o DE, e a Saúde Mental (SM), constituem a Componente Mental. Para além dos itens referidos, o SF-36 contempla ainda uma escala de Mudança de Saúde (MS) (item 2), que não faz parte de nenhuma das componentes, pois a resposta da pessoa a este item refere-se à perceção que esta tem acerca da mudança da sua saúde relativamente ao que acontecia há um ano atrás (Ferreira, 2000). Para a avaliação da perceção da QV há itens que são recodificados, de forma a que a valores maiores correspondam a uma perceção mais positiva. Os scores são obtidos pelo somatório dos itens, procedendo-se posteriormente a uma transformação para números índice (base 100). O instrumento apresenta uma boa consistência interna (valores de alfa de Cronbach variam entre 0,791 e 0,963).

A aplicação do questionário procedeu-se porta a porta, por entrevista e quanto à escolha dos respondentes adotou-se o método de Kish. Nos casos em que os selecionados se encontravam ausentes, sempre que possível procedeu-se a um agendamento posterior de forma a priorizar a resposta do próprio.
Na realização do presente estudo, os princípios éticos foram respeitados e orientaram todos os momentos desta investigação. Além disso, foi salvaguardada a privacidade dos indivíduos, pelo que os dados recolhidos foram tratados de forma a garantir o anonimato dos respondentes, bem como a confidencialidade dos dados.

Para a análise dos dados foram utilizadas medidas de estatística descritiva incluindo as distribuições de frequência, as medidas de tendência central e de dispersão, utilizando-se o programa Statistical Package for the Social Sciences (SPSS), versão 23.

\section{RESULTADOS}

\section{Caracterização Sociodemográfica}

Os resultados da caracterização sociodemográfica reportam-se às 603 pessoas incluídas no estudo. Quanto ao sexo, a população é maioritariamente do sexo feminino (56,2\%). A idade varia entre 5 meses e 99 anos, com média de $56,49 \pm 21,67$ anos e mediana de 62 anos. A faixa etária mais representada é a de 65 ou mais anos com $46,3 \%$. Em relação à coabitação, a população vive maioritariamente em famílias com agregados compostos por mais de 2 pessoas (50,2\%), sendo que $14,8 \%$ vivem sozinhas. No que concerne ao número de elementos que compõem o agregado familiar, este varia entre 1 e 8 , com média de 2,72 $\pm 1,27$ e mediana de 3 . A amostra é quase exclusivamente composta por pessoas de nacionalidade portuguesa, com $98,3 \%$. No que diz respeito ao estado civil, predominam os casados ou em união de facto $(56,1 \%)$, seguidos de $22,6 \%$ que são solteiros. Relativamente às habilitações literárias, $17,2 \%$ não frequentaram qualquer tipo de ensino e a maioria concluiu o $1^{\circ}$ ciclo do ensino básico $(46,4 \%)$. De referir ainda que $13,2 \%$ frequentaram o ensino pós-secundário ou superior. No que respeita à ocupação principal nas duas últimas semanas, verifica-se que a maioria dos inquiridos é reformada/aposentada $(38,5 \%)$, seguindo-se os que exercem uma profissão, têm um trabalho, mesmo não remunerado para uma pessoa da família (24\%). Considerando os dados relativos à profissão, a maioria inclui-se no grupo dos Trabalhadores qualificados da indústria, construção e artífices $(33,8 \%)$. Face à situação profissional verificase que $71,0 \%$ trabalha por conta de outrem, seguindose aqueles que trabalham por conta própria $(27,6 \%)$.

\section{Qualidade de Vida}

Os resultados relativos à Qualidade de vida são relativos às 447 pessoas que apresentavam critérios de inclusão para a sua avaliação. 
Analisando a tabela 1, constata-se que os valores médios mais elevados verificam-se na FS e DE, com médias de 84,4 $\pm 23,4$ e $82,0 \pm 24,5$, respetivamente. Em ambas as dimensões referidas, $50 \%$ ou mais da amostra têm uma perceção da FS e do DE que se encontra no limite máximo. Os valores mais baixos correspondem às dimensões SG $(51,6 \pm 20,1)$ e VT $(56,3$ $\pm 20,3)$. Nas dimensões FF, DF e DC, $25 \%$ ou mais da amostra têm uma perceção que se encontra no limite máximo. A QV total, decorrente de todas as dimensões apresentadas na tabela, varia entre o mínimo de 11,6 e o máximo de 95,9, com média de $68,6 \pm 18,7$, sendo que $50 \%$ ou mais das pessoas têm uma perceção de QV positiva.

Tabela 1 - Qualidade de Vida da população de Paredes de Coura $(\mathrm{n}=447)$

\begin{tabular}{|c|c|c|c|c|c|c|}
\hline \multirow{2}{*}{$\begin{array}{l}\text { Compo- } \\
\text { nente }\end{array}$} & \multirow{2}{*}{$\begin{array}{c}\text { Di- } \\
\text { mensão }\end{array}$} & \multirow{2}{*}{$\begin{array}{l}\text { Mín - } \\
\text { Máx }\end{array}$} & \multirow{2}{*}{$\begin{array}{c}\text { Média } \pm \\
\text { Desvio } \\
\text { Padrão }\end{array}$} & \multirow{2}{*}{$\begin{array}{l}\text { Medi- } \\
\text { ana }\end{array}$} & \multicolumn{2}{|c|}{ Percentis } \\
\hline & & & & & 25 & 75 \\
\hline \multirow{4}{*}{ Física } & $\mathrm{FF}$ & $0-100,0$ & $75,9 \pm 27,7$ & 85,0 & 55,0 & 100,0 \\
\hline & DF & $0-100,0$ & $75,8 \pm 27,7$ & 87,5 & 56,3 & 100,0 \\
\hline & DC & $0-100,0$ & $72,6 \pm 28,0$ & 74,0 & 52,0 & 100,0 \\
\hline & SG & $0-100,0$ & $51,6 \pm 20,1$ & 52,0 & 35,0 & 67,0 \\
\hline \multirow{5}{*}{ Mental } & SM & $0-100,0$ & $67,0 \pm 21,7$ & 70,0 & 55,0 & 85,0 \\
\hline & $\mathrm{DE}$ & $0-100,0$ & $82,0 \pm 24,5$ & 100,0 & 66,7 & 100,0 \\
\hline & FS & $0-100,0$ & $84,4 \pm 23,4$ & 100,0 & 75,0 & 100,0 \\
\hline & VT & $6,3-100,0$ & $56,3 \pm 20,3$ & 56,3 & 43,8 & 68,8 \\
\hline & $\begin{array}{l}\mathrm{QV} \\
\text { total }\end{array}$ & $11,6-95,9$ & $68,6 \pm 18,7$ & 72,6 & 56,2 & 83,9 \\
\hline
\end{tabular}

Relativamente à MS, a maioria da população considera que a sua saúde em comparação há um ano se mantém aproximadamente igual $(60,9 \%)$. Apenas $1,8 \%$ avalia a sua saúde como muito melhor.

\section{DISCUSSÃO}

No que concerne à faixa etária, analisando os dados com os obtidos pelo INE (2012), observa-se uma desigualdade mais evidente na faixa etária dos $65 \mathrm{ou}$ mais anos, pelo que se poderá concluir que há uma sobrerepresentação da população idosa e uma subrepresentação da população mais jovem. Esta particularidade pode trazer repercussões na perceção de QV desta população, tal como referido por Braga et al. (2011) e Praça (2012).

Os resultados obtidos neste estudo são corroborados pelos de Correia (2012) e aos de Fernandes (2014). Quanto ao estudo de Coelho e Pedroso (2012) os resultados são sobreponíveis à exceção da dimensão DC.
No estudo de Ayama, et al. (2009) os resultados são igualmente sobreponíveis, o que poderá estar relacionados com o facto da amostra de ambos ser predominantemente idosa, aliando-se às afirmações de Fechine \& Trompieri, em 2012 (Sousa, 2013). As dimensões da QV que apresentam melhores valores (DE e FS) estão inseridas na Componente Mental, o que também poderá estar relacionado com a elevada percentagem de idosos, tal facto é corroborado pelo estudo de Almeida (2013) e pelo de Coelho e Pedroso (2012). Atendendo a estes resultados, poder-se-á admitir que a população de Paredes de Coura apresenta uma melhor perceção do bem-estar psicológico e social, contrariamente à perceção do bem-estar físico. No que concerne aos resultados obtidos relativamente à FS, é de salientar que estes indicam que $25 \%$ ou mais da população realiza as suas atividades sociais normais sem interferência de problemas físicos ou emocionais (Louro, 2009 citando Pais-Ribeiro, 2005). Já em relação ao $\mathrm{DE}, 25 \%$ ou mais da população não tem dificuldades no trabalho ou nas atividades diárias como consequência de problemas emocionais (idem). Por outro lado, no que diz respeito à VT, os resultados indicam que $25 \%$ ou mais da população se sente cansada e exausta a maior parte do tempo (idem). Importa ainda referir os resultados relativos às dimensões FF e SM.

Na primeira constata-se que $50 \%$ ou mais da população apresenta valores positivos, sendo que, pelo menos $25 \%$ tem uma perceção da FF que se encontra no limite máximo. Estes valores indicam que a maioria da população realiza todo o tipo de atividade física sem limitações devido a motivos de saúde (idem). No que toca à SM, 50\% ou mais da população apresenta valores positivos, significando que metade ou mais da população se sente em paz, calma e feliz (idem), estando em conformidade com os resultados obtidos por Fernandes (2014). No que diz respeito à DC, menos de $25 \%$ da população apresenta valores baixos da perceção desta, o que indica que apenas uma pequena parte da população refere dor elevada e limitativa das atividades (Louro, 2009 citando Pais-Ribeiro, 2005). O estudo de Coelho e Pedroso (2012) opõe-se a estes resultados.

No presente estudo, a QV total, ou seja, a perceção que cada pessoa tem, apresenta valores tendencialmente positivos $(68,6 \pm 18,7)$, aproximando-se da realidade nacional (INE, 2009) e municipal (IMS, 2011; IMS, 2012a). 
Relativamente à MS, a população do Município de Paredes de Coura na sua maioria considera que, comparativamente há um ano atrás, a sua saúde mantémse aproximadamente igual. Estes resultados podem influenciar a perceção que a população tem da sua QV, segundo André (2005) e Martins (2006).

Quanto às limitações do estudo é de salientar que, as recusas em responder ao questionário, a ausência dos alojamentos ou as unidades de habitação não habitadas, poderão ter influenciado negativamente a representatividade da amostra. Espera-se que este estudo contribua para futuras investigações, de forma a contribuir para o melhor conhecimento do Perfil de Saúde do Alto Minho alargando, a outros Municípios. Também com o intuito de aprofundar o conhecimento sobre a QV de determinadas populações seria importante incluir o estudo de outras componentes objetivas e subjetivas. Assim, recomenda-se a utilização de um questionário em complementaridade ao SF-36. Finalmente, espera-se que os resultados sejam utilizados no sentido da melhoria da QV e da saúde da população de Paredes de Coura, bem como contribuir para alargar conhecimentos e fornecer bases a futuros estudos nesta mesma área e, por ventura, nesta mesma comunidade.

\section{CONCLUSÕES}

A QV é influenciada por uma variedade de fatores que vão para além da saúde de cada indivíduo, incluindo várias condições que afetam a perceção individual. Tendo em conta o quadro de referências, e dando resposta aos objetivos delineados, concluiu-se que:

- A população do Município de Paredes de Coura é maioritariamente idosa, com predomínio do sexo feminino e com um baixo nível de escolaridade;

- A perceção da qualidade de vida é globalmente positiva. Os melhores valores são relativos às dimensões Desempenho Emocional e Função Social que estão inseridas na Componente Mental, o que denota que a população de Paredes de Coura apresenta melhor perceção do bem-estar psicológico e social na sua QV, que na Vitalidade e Saúde Geral, pertencentes à Componente Mental e Física, onde se observa pior perceção;

- Na sua maioria a população considera que, comparativamente há um ano atrás, a sua saúde mantém-se aproximadamente igual.

Em síntese, a população do Município de Paredes de Coura, na sua globalidade, apresenta uma perceção de QV positiva, destacando-se a Componente Mental.

\section{IMPLICAÇÕES PARA A PRÁTICA CLÍNICA}

Para a Enfermagem estes estudos revelam-se importantes na medida em que permitem o acesso a mais informação em saúde, bem como das dimensões que mais afetam positiva ou negativamente a sua QV. Deste modo, torna-se possível que os profissionais de saúde possam orientar melhor a sua ação através de estratégias de intervenção mais sustentadas e adequadas com vista a produzirem mais ganhos em saúde e QV.

\section{REFERÊNCIAS BIBLIOGRÁFICAS}

Almeida, M. C. B. M. (2013). Motivação e Comportamentos de Saúde: Relação com a Qualidade de Vida em Adultos da Comunidade. Tese de Doutoramento, Universidade do Porto, Faculdade de Psicologia e Ciências da Educação, Porto, Portugal.

André, C. M. (2005). Qualidade de Vida e Doença Coronária. Coimbra, Portugal: Formasau - Formação e Saúde, Lda.

Ayama, S., Rodrigues, L. A., Silva, R. B. L. e Feriancic, M. M. (2009). Análise da Qualidade de Vida e Independência em Octogenários. III Congresso Iberoamericano de Psicogerontologia.

Braga, S. F. M., Peixoto, S. V., Gomes, I. C., Acúrcio, F. A., Andrade, E. I. G., e Cherchiglia, M. L. (2011). Fatores associados com a qualidade de vida relacionada à saúde de idosos em hemodiálise. Revista Saúde Pública, 45 (6), 1227-1236. doi.org/10.1590/S003489102011000600015

Coelho, E. S. P., e Pedroso, M. F. B. P. (2012). Avaliação da Qualidade de Vida em Idosos Residentes no Município de Santos / SP. Revista Ceciliana, 1 (4), 4-8.

Correia, C. S. L. (2012). A Qualidade de Vida e a Solidão em idosos institucionalizados e na comunidade. Tese de Mestrado, Instituto Superior de Psicologia Aplicada, Lisboa, Portugal.

Fernandes, F. J. F. (2014). "Perceção do estado de saúde da população idosa do Alto Minho: um contributo para o planeamento em saúde". Tese de Mestrado, Instituto Politécnico de Viana do Castelo, Viana do Castelo, Portugal. 
Ferreira, P. L. (1998). A Medição do Estado de Saúde: Criação da Versão Portuguesa do MOS SF-36. Coimbra, Portugal: Centro de Estudos e Investigação em Saúde da Universidade de Coimbra.

Ferreira, P. L. (2000). Criação da versão Portuguesa do MOS SF-36: Parte I - Adaptação cultural e linguística. Acta Médica Portuguesa, 13, 55-66.

Gonçalves, J. D. A. (2008). Percepção de saúde e qualidade de vida dos enfermeiros. Tese de Mestrado, Instituto Politécnico de Beja, Beja, Portugal.

IMS. (2011). Síntese de resultados das freguesias da Areosa, Meadela e Santa Maria Maior. Relatório de Investigação, Escola Superior de Saúde, Viana do Castelo, Portugal.

IMS. (2012a). Perfil de Saúde da População de Darque Cuidados Preventivos, Insegurança Alimentar e Qualidade de Vida - Contributos para o Observatório de Saúde. Relatório de Investigação, Escola Superior de Saúde, Viana do Castelo, Portugal.

INE. (2009). Inquérito Nacional de Saúde 2005/2006. Lisboa, Portugal: Instituto Nacional de Saúde Doutor Ricardo Jorge.

INE. (2012). Censos 2011. Lisboa, Portugal. Acedido em http://mapas.ine.pt/map.phtml.
Lourenço, L. M. P. (2013). A Influência da Pobreza no Estado de Saúde da População Idosa de Paredes de Coura. Tese de Mestrado, Instituto Politécnico de Viana do Castelo, Escola Superior de Saúde, Viana do Castelo, Portugal.

Louro, S. P. (2009). O impacto da incapacidade física na qualidade de vida relacionada com a saúde nos doentes oncológicos: um estudo exploratório. Tese de Mestrado, Universidade de Lisboa, Faculdade de Psicologia e Ciências da Educação, Lisboa, Portugal.

Martins, T. (2006). Acidente Vascular Cerebral - Qualidade de vida e bem-estar dos doentes e familiares cuidadores. Coimbra, Portugal: Formasau - Formação e Saúde, Lda.

Praça, M. I. F. (2012). Qualidade de vida relacionada com a saúde: a perspectiva dos utentes que frequentam os Centros de Saúde do ACES Trás-os-Montes I Nordeste. Tese de Mestrado, Instituto Politécnico de Bragança, Bragança, Portugal

Sousa, J. P. (2013). Qualidade de vida do idoso: Um estudo de revisão. Porto, Portugal: Universidade Fernando Pessoa, Faculdade de Ciências do Porto.

Zamberlan, C., Calvetti, A., Deisvaldi, J., \& De Siqueira, HCH. (2010). Qualidade de vida, saúde e enfermagem na perspectiva ecossistêmica. Revista Enfermeria Global, 20, 1-7. Acedido em http://scielo.isciii.es/pdf/ eg/n20/pt_reflexion2.pdf 